\title{
Digital Medical Image Segmentation Using Fuzzy C-Means Clustering
}

\author{
Bakhtyar Ahmed Mohammed', Muzhir Shaban Al-Ani² \\ ${ }^{1}$ Department of Computer Science, University of Human Development, College of Science and Technology, \\ Sulaymaniyah, KRG, Iraq, ${ }^{2}$ Department of Information Technology, University of Human Development, College of \\ Science and Technology, Sulaymaniyah, KRG, Iraq
}

\section{A B S T R A C T}

In the modern globe, digital medical image processing is a major branch to study in the fields of medical and information technology. Every medical field relies on digital medical imaging in diagnosis for most of their cases. One of the major components of medical image analysis is medical image segmentation. Medical image segmentation participates in the diagnosis process, and it aids the processes of other medical image components to increase the accuracy. In unsupervised methods, fuzzy c-means (FCM) clustering is the most accurate method for image segmentation, and it can be smooth and bear desirable outcomes. The intention of this study is to establish a strong systematic way to segment complicate medical image cases depend on the proposed method to share in the decision-making process. This study mentions medical image modalities and illustrates the steps of the FCM clustering method mathematically with example. It segments magnetic resonance imaging (MRI) of the brain to separate tumor inside the brain MRI according to four statuses.

Index Terms: Medical image, Medical image modality, Segmentation, Fuzzy C-means clustering

\section{INTRODUCTION}

The interest is shown by medical professionals in deepening their knowledge of internal anatomy plays an essential part in the importance of medical images that are used in both treatment and diagnosis [1]. Numerous methods of diagnostic medical imaging have been created dependent on different types of electromagnetic band imaging which includes gamma-ray and X-ray imaging, cross-sectional pictures, such as computed tomography (CT), single-photon emission CT, positron emission tomography, magnetic resonance imaging $(\mathrm{MRI})$, or ultrasound [1,2]. These various

\begin{tabular}{|l|l|}
\hline \multicolumn{2}{|c|}{ Access this article online } \\
$\begin{array}{ll}\text { DOI: 10.21928/uhdjst.v4n1y2020.pp51-58 } & \text { E-ISSN: 2521-4217 } \\
\text { P-ISSN: 2521-4209 }\end{array}$ \\
\hline
\end{tabular}

Copyright (C) 2020 Mohammed and Al-Ani. This is an open access article distributed under the Creative Commons Attribution NonCommercial No Derivatives License 4.0 (CC BY-NC-ND 4.0) applications and techniques in medical image processing rely on different ranges of electromagnetic spectrum bands [2]. Improvements in technology caused to increase size and volume of medical imaging, also these developments raise demand on automated diagnosis with computer technology developments, also it decreases the cost and time [3].

Image segmentation can be described as; trying to find homogenous limits inside an image and after that the classification of them, and can be considered as the most significant field of medical image processing, also it allows images to be divided into relevant areas according to homogeneity or heterogeneity criteria, and it is an automatic or semi-automatic process used for separating the region of interest (ROI), also there are numerous medical applications that use to differentiate in the segmentation of body organs and tissues, these include cardiology image analysis, breast tumor detection, autoclassification in hematology field, brain cognitive development, mass segmentation, mass detection, 
surgery simulations, plan of surgery, and detection of vessel boundary in coronary angiograms $[1,4]$. Techniques of image segmentation can be characterized according to these essential terminologies, such as oriented of pixel, color, region, model, and hybrid [4]. Intelligent decision support systems commonly use the prevailing image segmentation to accurately organize image pixels [5]. The procedure divides the picture into systematic and defined sectors depending on their similarities [5]. Image segmentation is one main component of analysis processes which use in these techniques; remote sensing, computer vision, medical image processing, and geographical information system [6]. Image segmentation plays a key role in automated object recognition systems in the process of computer vision and medical imaging for the analysis of details, image segmentation enables greater ease in detecting and quantifying abnormalities in anatomical structures, such as the brain and lung [5].

However, the processing of the image segmentation can be affected by improper illumination, noise disturbances, environmental factors, and blurring of images, an important phase toward the automatic segmentation of images is region segmentation since this is the step taken to determine and segment the area of interest [7]. Because of ease to apply fuzzy c-mean (FCM) and its high accuracy, it has become one of the best way for image segmentation [8]. Nevertheless, FCM has inadequacies in confusion acknowledgment; various undertakings are practiced for covering this deficiency, with using the objective work FCM and the use of neighbor pixels despite the pixel and besides pixel division have been used, FCM methodology is used for improving the accuracy in picture division, enlistment work is changed [8].

The best criterion to find the optimum solution for these issues is the method known as FCM clustering which is a clustering method whereby points of data can be designated to more than one group each based on shared correlations, and then tries to identify parallels and relationships within each set, least-squares solutions are employed to identify the ideal location for any point of data which may lie in a space of probability bounded by two or more clusters, also there should be as higher level as possible in the likeness of clusters and as lower-level as possible in differences and fuzzy boundaries are easier to develop from a computational point of view [9]. The purpose of writing this paper is to indicate the importance of the segmentation process in image processing and the mechanism of brain segmenting image using FCMs clustering and how it can find the optimum solution for segmenting images and diagnosis using medical image techniques. Furthermore, there are four major steps in the medical imaging field, which consist of capturing the image, digitalizing it, processing for segmentation and finally extracting important information $[9,10]$.

\section{LITERATURE REVIEW}

In 2008, Ahmed and Mohamad explored that fuzzy clustering is very important in image segmentation, using parallel length to calculate fuzzy weights [11].

In 2010, Naz et al. found that the reason digital image processing developers are innovating this method is the best and most accurate way of diagnosing medical imaging can be found, improvements have been made rapidly with many methodologies being put forward supported by a wide range of literature on taking information from a picture and dividing it into defined areas. However, constraints are presented in regards to intricacy, time, and precision as a result of unclear cluster borders shown in images, fuzzy techniques, on the other hand, are largely free of such problems and provide much better results in comparison to other segmented image methods [6].

In 2010, Padmavathi et al. stated that quality of underwater image is different from the quality of an image which capture in air, because some factors have impact of it such as; water medium, atmosphere, pressure, and temperature which means that image segmentation is necessary for digital image processing that implies image demonstrations is the need of picture segmentation, which separates a picture into portions that have strong correlations with objects to mirror the genuine data gathered from this present reality, picture segmentation is the most down to earth approach among practically all robotized picture acknowledgment frameworks, also clustering of numerical information shapes the premise of numerous arrangement and framework displaying calculations [7].

In 2011, Quintanilla-Dominguezab et al. tested FCM for the early stages of breast cancer detection using mammography technique [12].

In 2013, Jiang et al. utilized the fuzzy science strategy, and fuzzy grouping examined isolates the differentiate things and arranges them [13].

Furthermore, In 2013, Yambal and Gupta showed that segmentation process is unsupervised classification technique and an important step in advance image analysis process 
used as assistance to some other processes like detection for MRI brain tumor, generally original works of clustering are detecting anomalies, identifying salient features, classifying data, and compressing data, using conventional FCMs algorithm depend on hierarchical self-organized map, the aim of image segmentation process is to effective segmentation of noisy images [14].

In 2014, Khalid et al. illustrated that FCMs can diagnose some special diseases, such as glaucoma which is an ailment characterized by expanded weight inside the eyeball, making extreme harm the optic nerve, it is the most astounding reason for visual deficiency and irreversible, with early revelation early and appropriate treatment which it could continue for a long time [15].

Furthermore, In 2014, Norouzi et al. showed that mechanism of clustering algorithms is same as the classification technique without training of data, these methods have unsupervised learning algorithm and individual authority to calculate the similar features in the image and retain some things, same as; keys to recognize other features that have same attributes, this method is compatible with most of the data mining algorithms considering unsupervised methods they do not train data because its process is not time consuming in segmenting [1].

In 2017, Kumar et al. tested correlative distance by adding the process of eliminating, clustering, and merging to compute fuzzy weights using large initial prototypes and Gaussian weights. In standard FCM spatial FCMs methods incorporated the spatial information and altering of every cluster membership weights after considering the cluster distribution in the neighborhood [3].

In 2018, Ali et al. denoted that segmentation is an essential step to the sensitive analysis of human tissue lesions with aim of improving the partition of different clusters of images rely on similar features [16].

\section{MEDICAL IMAGE MODALITIES}

Imperative topic in medical imaging is medical image modalities or techniques. It is used to anatomical vision of body organs. There are some modalities which use in digital medical image processing.

\subsection{X-Ray}

Nowadays, X-ray imaging system uses in the branch of diagnostic radiology, however, there are many types of $\mathrm{X}$-ray imaging systems, but the most famous one is conventional planar radiography which is too beneficial because of its low cost and low dose with side effect of interacting with useless beams. Its problem is overlapping anatomical image details and finding a lot of clusters [17].

In telemedicine uses as an integral part to automate X-ray segmentation which use broadly in remote areas which usually accidents happen there which helps the medical staffs to analyze the emergency cases, in this situation X-ray analysis in two manners; first one is segmenting the bone region from the surrounding flesh of the bone then extraction their features, the other one is determining the situation of the bone which usually happen this case, also it automatically selecting fractions aim doctors and medical staffs to analyze the level of injury to select the suitable medical treatment [17].

\subsection{CT}

To avoid overlapping effect, CT has been evolved. It can reshape the picture from all sides and angles until it can recreate the image of the body organ. High radiation dose is side effect of CT, also side effect of using that big amount of radiation increased day after day until it has detected that using CT cause of cancer higher than other types [17].

\subsection{Digital Tomosynthesis}

This technique has been invented to solve the problems of overlapping and high dose of radiation. It has moderate performance between these two disadvantages of X-ray and CT. Its angle was $<360$, but in digital theater systems, resolution depth increased with lower radiation compared to CT, because of this advantage, little angles accurately reshape images. Two major reshaping methods have been evolved: Analytical reconstruction and iterative reconstruction. In different algorithms of analytical reconstruction, filtered back-projection was the most famous one because of its smallest deforming and high precision. However, this method needs high pass filtering, to prevent this distortion maximum likelihood expectation maximization (MLEM) evolved which essentially involved iteration number with edge-preserving regularization. Another algorithm invented based on MLEM known as chest digital tomosynthesis (CDT) system to project data using a real CDT system [17].

\subsection{MRI}

Using of MRI images more common than other modalities in brain diagnosis and segmentation process. Among unsupervised learning methods FCMs clustering for medical image segmentation methods, FCM clustering is the most accurate one for segmentation process compare to other segmentation methods more appropriate for sensitivity 
noisy with intensity inhomogeneity MRI which can properly decrease the noises and supply superior segmentation results [18].

MRI is a wide medical modality that takes the image of the internal body and organs without contacting the skin. The characteristics of MRI are non-linear. A lot of things have influences of MRI accuracy, such as partial volumes effects which implies a pixel consists of more than one tissues, also, the volume is dependable thing in the segmentation process so as to determine the size of organs and strange things [19].

\subsection{High-resolution CT}

The segmentation is an indispensable step, as in many medical image analysis applications. Accurate segmentation using high-resolution CT (HRCT) images and quantification of the lungs have an important role in the early diagnosis of lung diseases. Especially detection of nodules in the lung region, airway and vessels diameter, lung volume is the key component of diagnosing lung diseases. The definition of each lung region from the CT images is the first step for the computer-aided diagnosis algorithm of lung. To extract each lung tissue, FCMs clustering algorithm has been applied to the segmentation of lung region in two-dimensions HRCT images [5].

\section{METHODOLOGY}

Many methods developed during several past years to enhance medical segmentation with demanding to obtain an accurate diagnosis, but FCM was the most accurate one in unsupervised learning methods. In supervised learning, when you feed the images or specified dataset to the method, it can extract similar features and cluster them according to observe of patterns. FCM distribute one piece of data to two or more clusters. However, the con of these methods is cannot label similar groups. For instance, in the tested image, FCM can determine the tumor without labeling it.

FCM clustering is the most accurate and widely method in the segmentation process of digital medical image processing compared to other methods. It acts as preprocessing, which aids classification and detection processes and sometimes use as a diagnosis process. Mentioning of fuzzy k-means (FKM) clustering is important because it used before FCMs clustering. Nowadays, using FCM is wider than FKM clustering, because it could solve these problems that had been happened in FKM clustering.
FKM creates segmentations hierarchically, which involve each data point that can only be specified in one cluster, but FCM permits data points to be allocated into more than one cluster in which each data point has a degree of the endurance of belonging to each cluster as in fuzzy logic.

The mechanism of the process pass through these steps, as shown in Fig. 1; the first step is importing an MRI image that captured according to the specific technique of electromagnetic band imaging after that is converting the image in analog to same digital image size inside data acquisition step. Then preprocessing image begin by preparing images to the segmentation process, which involves image denoising and restoration. The process used median filter, as shown in Fig. 2. Then, FCM clustering implement and segment images into four parts. After that, the process converts the same image to same size analog image. Finally, export segmented image is as shown in Fig. 3.

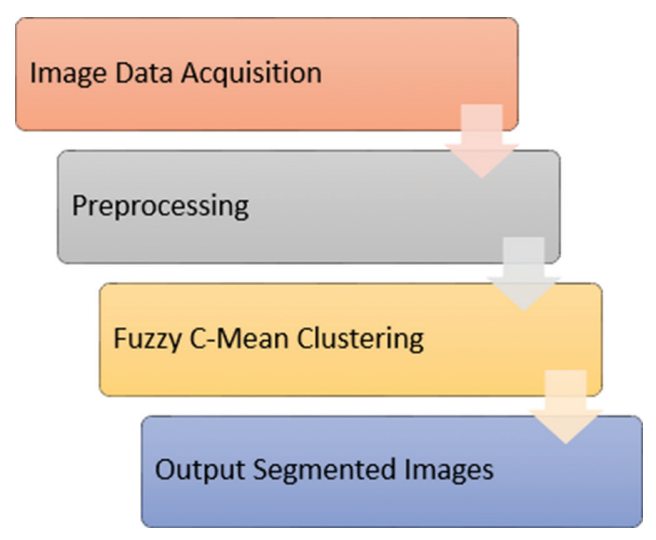

Fig. 1. Processes of FCM clustering.

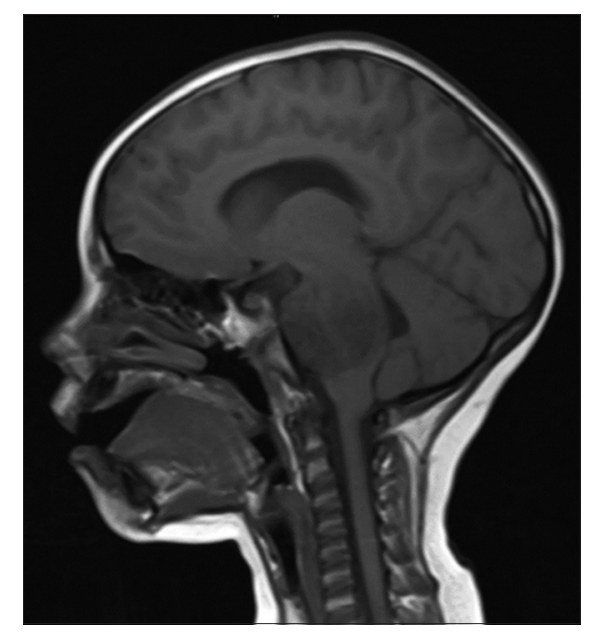

Fig. 2. Original image after the filtration process. 


\subsection{Mathematical Model of FCMs Clustering}

In general, core of the FCM method is a mathematical model which relies on five steps until it can cluster similar features together inside one region. Because the first step of the work starts by taking values randomly from the membership matrix, so this example has depended on the imaginary value of two-dimensional image that illustrates the mathematical process of FCM clustering.

*m: fuzzification parameter; its range between (1.25 and 2).

while $\mathrm{m}: 2$, i: first data point, j: first cluster, c: number of clusters.

\subsubsection{First step}

Randomly initialized the values as the membership matrix (Um) to the original image according to Equation.1, number of objects is 8 , number of clusters is 4 , The fuzziness parameter range $(\mathrm{m})$ between (1.2 and 2), as shown in Table 1. Using this Equation 1.

$$
\sum_{(j=1)}^{c} \mu_{j}\left(x_{i}\right)=1 \ldots \ldots i: 1,2,3,4, \ldots \ldots ., \mathrm{k}
$$

\subsubsection{Second step}

After that, the model find constraints for every cluster. According to Equation 2.

$$
C_{-j}=\frac{\sum_{i}\left(\mu_{j}(X i)\right)^{m} X_{i}}{\sum_{i}\left(\mu_{j}(X i)\right)^{m}}
$$

\subsubsection{Third step}

After that, the model finds distance (Di) for every cluster and find centroids, for instance, in the current example find; centroid (1), centroid (2), centroid (3), and centroid (4), using Euclidean rule, according to the Equation 3.

$$
\mathrm{D}_{\mathrm{i}}=\sqrt{\left(\mathrm{x}_{2}-\mathrm{x}_{1}\right)^{2}+\left(\mathrm{y}_{2}-\mathrm{y}_{1}\right)^{2}}
$$

After that, the model finds distances for every cluster, as shown in Table 2.

\subsubsection{Fourth step}

Updating the membership values, according to the Equation 4.

$$
\mu_{j}\left(x_{i}\right)=\left(\frac{1}{d_{j i}}\right)^{\frac{1}{m-1}} / \sum_{k=1}^{c}\left(\frac{1}{d_{k i}}\right)^{\frac{1}{m-1}}
$$

In this case, $m=2, i=$ first data point, $j$ =first cluster, it is important new cluster values and put them in the new table so as to compare them with earlier cluster values to get new membership values, as shown in Table 3;

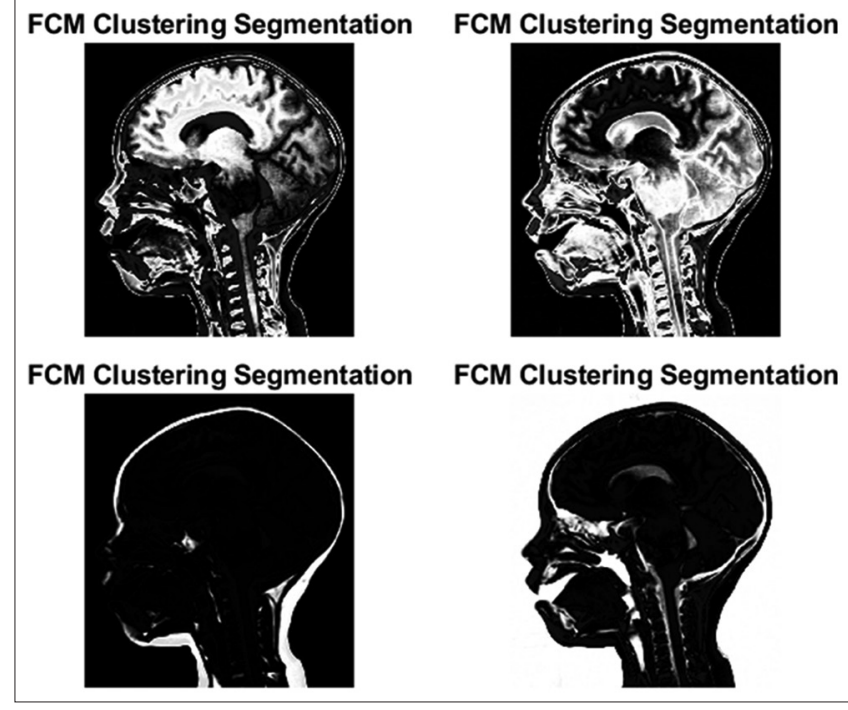

Fig. 3. All processes together.

TABLE 1: $X$ and $Y$ values of eight objects with initial values of four clusters of them

\begin{tabular}{cccccc}
\hline $\mathbf{X}$ & $\mathbf{Y}$ & $\mathbf{C 1}$ & $\mathbf{C 2}$ & $\mathbf{C 3}$ & $\mathbf{C 4}$ \\
\hline 2 & 8 & 0.1 & 0.2 & 0.3 & 0.4 \\
4 & 6 & 0.3 & 0.4 & 0.6 & 0.8 \\
6 & 4 & 0.5 & 0.6 & 0.9 & 0.2 \\
8 & 2 & 0.7 & 0.8 & 0.2 & 0.6 \\
1 & 7 & 0.2 & 0.1 & 0.5 & 0.1 \\
3 & 5 & 0.4 & 0.3 & 0.8 & 0.3 \\
5 & 3 & 0.6 & 0.5 & 0.1 & 0.5 \\
7 & 1 & 0.8 & 0.7 & 0.4 & 0.7 \\
\hline
\end{tabular}

\subsubsection{Final step}

This process iterates until it arrives correct centroids.

\subsection{FCMs Algorithm}

- Initialize the membership matrix of randomize values (fuzziness partition)

- Calculate the centroid vectors using the following equations

$$
C j=\frac{\sum_{i}\left(\mu_{j}(X i)\right)^{m} X_{i}}{\sum_{i}\left(\mu_{j}(X i)\right)^{m}}
$$

And

$$
\mathrm{D}_{\mathrm{i}}=\sqrt{\left(\mathrm{x}_{2}-\mathrm{x}_{1}\right)^{2}+\left(\mathrm{y}_{2}-\mathrm{y}_{1}\right)^{2}}
$$

- Update partition matrix for new elements according to this equation

$$
\mu_{j}\left(x_{i}\right)=\left(\frac{1}{d_{j i}}\right)^{\frac{1}{m-1}} / \sum_{k=1}^{c}\left(\frac{1}{d_{k i}}\right)^{\frac{1}{m-1}}
$$


Mohammed and Al-Ani: Digital Medical Image Segmentation Using FCM Clustering

TABLE 2: Data point and distance for all clusters

\begin{tabular}{|c|c|c|c|c|c|c|c|}
\hline \multicolumn{2}{|c|}{ Cluster 1} & \multicolumn{2}{|c|}{ Cluster 2} & \multicolumn{2}{|c|}{ Cluster 3} & \multicolumn{2}{|c|}{ Cluster 4} \\
\hline Data point & Distance & Data point & Distance & Data point & Distance & Data point & Distance \\
\hline$(2,8)$ & 6.79 & $(2,8)$ & 6.764 & $(2,8)$ & 3.84 & $(2,8)$ & 5.385 \\
\hline$(4,6)$ & 4 & $(4,6)$ & 3.954 & $(4,6)$ & 1.065 & $(4,6)$ & 2.59 \\
\hline$(8,2)$ & 1.93 & $(8,2)$ & 1.84 & $(8,2)$ & 4.675 & $(8,2)$ & 3.187 \\
\hline$(1,7)$ & 6.76 & $(1,7)$ & 6.79 & $(1,7)$ & 4.223 & $(1,7)$ & 5.416 \\
\hline$(3,5)$ & 3.95 & $(3,5)$ & 4 & $(3,5)$ & 2.05 & $(3,5)$ & 2.656 \\
\hline
\end{tabular}

\section{TABLE 3: Manually solved values of clusters}

\begin{tabular}{cccccc}
\hline $\mathbf{X}$ & $\mathbf{Y}$ & Cluster 1 & Cluster 2 & Cluster 3 & Cluster 4 \\
\hline 2 & 8 & 0.198689327 & 0.199453065 & 0.351328263 & 0.250529346 \\
4 & 6 & 0.136763286 & 0.138354361 & 0.513664923 & 0.21121743 \\
6 & 4 & 0.204349796 & 0.225947742 & 0.148618034 & 0.421084428 \\
8 & 2 & 0.326016177 & 0.34196262 & 0.134590635 & 0.197430568 \\
1 & 7 & 0.206419945 & 0.205507927 & 0.330428327 & 0.257643801 \\
3 & 5 & 0.185132797 & 0.182818637 & 0.356719292 & 0.275329273 \\
5 & 3 & 0.26436788 & 0.23909742 & 0.127020505 & 0.369514195 \\
7 & 1 & 0.346019973 & 0.329884326 & 0.127590531 & 0.19650517 \\
\hline
\end{tabular}

- Repeating this process by checking convergence until it gets the same manually result in centroids if not the loop returns back to step 2 .

\section{RESULTS AND DISCUSSION}

The imperative thing in the procedure of digital medical image segmentation is the sensor or camera which use to capture images. This steps the primary step in the process and it has direct interaction with the physical organic things because the natural form of every signal is an analog signal. In medical imaging, it varies according to techniques of electromagnetic band imaging because any technique uses in the specified range to take an image. This study relied on using MRI because it is the most effective technique to diagnose brain tumor. Most of the time converting analog to digital happen inside the sensor. Data acquisition is the first and important process in methodology for the training and testing process, but FCM clustering method is unsupervised learning. Testing on this method did not depend on the specified dataset because it is only segmentation process and tested on five ready MRI images taken from the internet that properly applied on the proposed method, which could clustered images well, as shown in Fig. 4. After that, preprocessing step begins by image restoration using median filter and resizing because the segmentation process needs to find regions accurately and median filter does same process

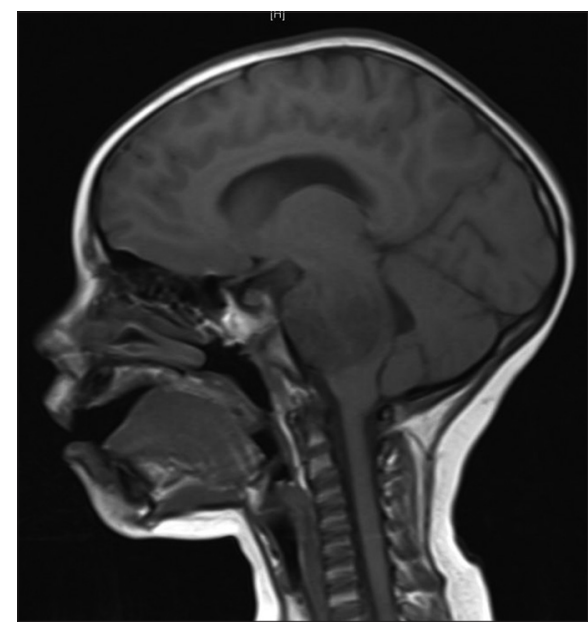

Fig. 4. Original image.

without damaging any edges. The next step is implementing FCMs algorithm which use to segmenting medical images accurately. The proposed method dispatches the images to some clusters according to areas and how much iteration necessary to execute the method until it can show the accurate results. The backbone of the issue is the results of the algorithm that is applied on and original gray scale image of 4 years child head MRI image in the left side. The target of this process is diagnosing an abnormal mass in the brain, as shown in Fig. 4.

The best method to solve these cases is FCM clustering because it simplifies the process of feature extraction. It separates different attributes according to these clusters that determine optionally. For instance, in the current method determined four clusters. The FCN algorithm depends on the $\mathrm{fcn} 0$ method inside fuzzy logic toolbox inside the MATLAB tool. Every mathematical step automatically happens inside the ready method according to the called image, but it iterates inside the method automatically 100 times to find the values of fcn objects and cluster them. Matrix Laboratory known as MATLAB is a practical robust tool used to conduct 
procedures within the processing of digital images. It used to carry out mathematical computations with matrices and vectors, which is very straightforward to operate as it incorporates computation, visualization, and programming inside the system [21].

Many methods used to prevent the noise problem in digital image processing. However, the best filtering technique in this case is median filtering. In addition, different types of filters are applied to remove different types of noise. There are many types of filtering, such as median, mean (average), and Gaussian.

After that, implementing the FCM clustering method starts by applying preprocessed images to the FCM method. It involves grouping the similar images data and distributing it into $\mathrm{N}$ clusters with all data points inside the images.

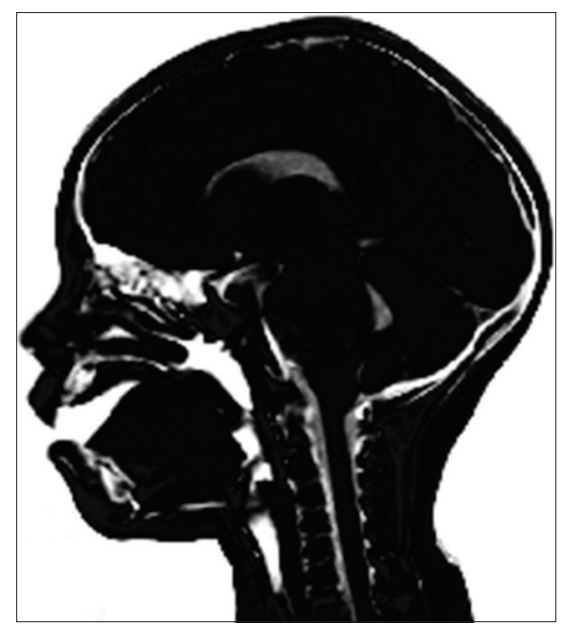

Fig. 5. Spaces inside the head magnetic resonance imaging.

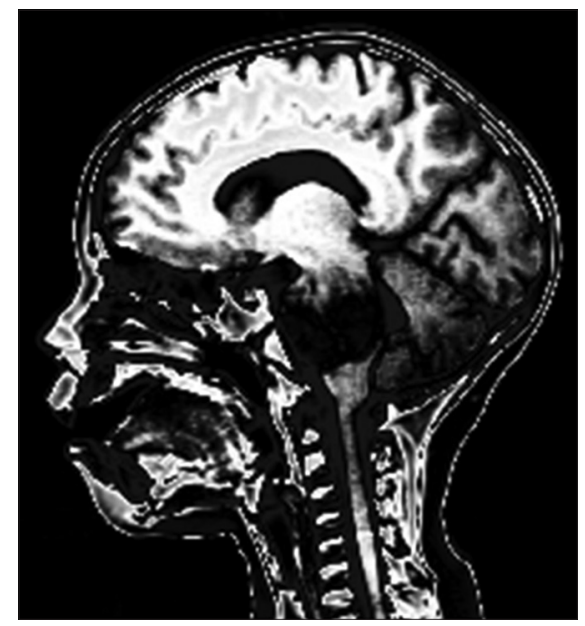

Fig. 6. Segmented tumor image.
Different clusters exhibit different visions, as shown in Fig. 5. This method can illuminate some properties of the image. It makes clusters over that rule.

Fig. 6 shows the mass inside the head and it aids the medical professionals to decide accurately.

Fig. 7 shows the image separated into $\mathrm{N}$ clusters.

Fig. 8 shows the background of the image.

In general, all of these processes mixed in this method, as shown in Fig. 3.

The result of FCM is the most accurate compared to other unsupervised learning methods. FCM is used in a wide range of applications compared to other types, especially

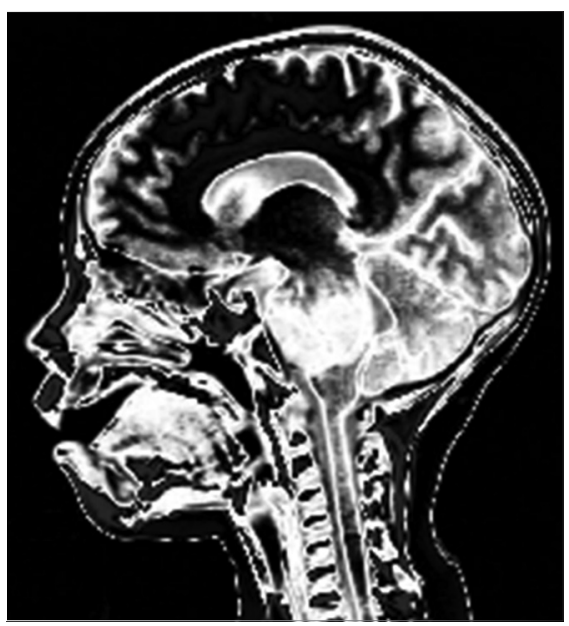

Fig. 7. Segmentation according to $\mathrm{N}$ clusters.

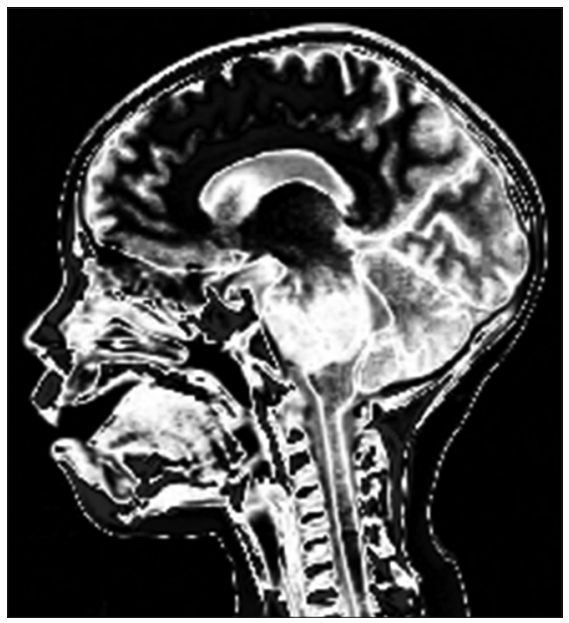

Fig. 8. Background of the image. 
in diagnosing. Recently, it uses in most of the segmentation cases relate to medical images.

\section{CONCLUSIONS}

New image segmentation process using FCM clustering is very important to get every desired feature and making clusters to extract their patterns. In addition, the results of every step are very important for manually working to find what are the weak points capable to change and improve. Comparison between final values to initial values is done to realize the difference between them. The FCM clustering method performs this process automatically, but changing parameters are so beneficial criterion to get the best accurate segmented images.

FCM method is the most effective segmentation approach in unsupervised learning methods which used in medical image segmentation processes. The mathematical procedure of this approach is illustrated step by step. FCM clustering is an unsupervised learning method, which is an accurate segmentation process. It can rely in on diagnosis process, such as brain tumor, which tested. Then, the important role in finding the boundary of components of medical images and how it can find ROI and segment the organs and abnormal shapes. This approach supports medical professionals to take the correct decision.

\section{REFERENCES}

[1] A. Norouzi, M. S. M. Rahim, A. Altameem, T. Saba, A. E. Rad, A. Rehman and M. Uddin. "Medical image segmentation methods, algorithms, and applications". IETE Technical Review, vol. 31, no. 3, pp. 199-213, 2014.

[2] R. C. Gonzalez and R. E. Woods. "Digital Image Processing". $4^{\text {th }}$ ed. Pearson Education, New York, 2018.

[3] R. Kumar, G. Satheesh and B. Nisha. "MRI brain image segmentation using Fuzzy C means cluster algorithm for tumor area measurement". International Journal of Engineering Technology Science and Research, vol. 4, no. 9, pp. 929-935, 2017.

[4] T. Saikumar, P. Yugander, P. S. Murthy and B. Smitha. "Colour Based Image Segmentation Using Fuzzy C-Means Clustering". In: International Conference on Computer and Software Modeling, Singapore, 2011.

[5] E. Doğanay, S. Kara, H. K. Özçelik and L. Kart. "A hybrid lung segmentation algorithm based on histogram-based fuzzy C-means clustering". Journal Computer Methods in Biomechanics and Biomedical Engineering: Imaging and Visualization, vol. 6, no. 6, pp. 638-648, 2014.

[6] S. Naz, H. Majeed and H. Irshad. "Image Segmentation Using Fuzzy Clustering: A Survey". In: $6^{\text {th }}$ International Conference on
Emerging Technologies, Islamabad, Pakistan, 2010.

[7] G. Padmavathi, M. M. Kumar and S. K. Thakur. "Nonlinear image segmentation using fuzzy c means clustering method with thresholding for underwater images". IJCSI International Journal of Computer Science Issues, vol. 7, no. 3, pp. 35-40, 2010.

[8] O. Jamshidi and A. H. Pilevar. "Automatic segmentation of medical images using Fuzzy c-means and the genetic algorithm". Journal Computational Medicine, vol. 2013, p. 972970, 2013.

[9] G. Stephanie. "Fuzzy Clustering Definition", 2016. Available from: https://www.statisticshowto.datasciencecentral.com/fuzzyclustering. [Last accessed on 2019 Oct 01].

[10] L. Ma, H. Chen, K. Meng and D. Liu. "Medical Image Segmentation Based on Improved Fuzzy C-Means Clustering". In: International Conference on Smart Grid and Electrical Automation, Changsha, China, 2017.

[11] M. M. Ahmed and D. B. Mohamad. "Anisotropic diffusion model segmentation of brain MR images for tumor extraction by combining kmeans clustering and Perona-Malik". International Journal of Image Processing, vol. 2, no. 1, pp. 27-34, 2008.

[12] J. Quintanilla-Dominguezab, B. Ojeda-Magañaac, M. G. CortinaJanuchsab, R. Ruelasc, A. Vega-Coronab and D. Andinaa. "Image segmentation by fuzzy and possibilistic clustering algorithms for the identification of microcalcifications". Scientia Iranica, vol. 18, no. 3, pp. 580-589, 2011.

[13] H. Jiang, Y. Liu, F. Ye, H. Xi and M. Zhu. "Study of clustering algorithm based on Fuzzy C-means and immunological partheno genetic". Journal of Software, vol. 8, no. 1, p. 134, 2013.

[14] M. Yambal and H. Gupta. "Image segmentation using Fuzzy C means clustering: A survey". International Journal of Advanced Research in Computer and Communication Engineering, vol. 2, no. 7, pp. I-5, 2013.

[15] N. E. A. Khalid, N. M. Noor and N. Ariff. "Fuzzy c-means (FCM) for optic cup and disc segmentation with morphological operation". Procedia Computer Science, vol. 42, pp. 255-262, 2014.

[16] N. A. Ali, B. Cherradi, A. E. Abbassi, O. Bouattane, M. Youssfi. "GPU Fuzzy c-means algorithm implementations: Performance analysis on medical image segmentation". Multimedia Tools and Applications, vol. 77, no. 16, pp. 21221-21243, 2018.

[17] O. Bandyopadhyaya, A. Biswasa and B. B. Bhattacharyaba. "Long-bone fracture detection in digital X-ray images based on digital-geometric techniques". Computer Methods and Programs in Biomedicine, vol. 123, pp. 2-14, 2016.

[18] S. K. Adhikari, J. K. Sing, D. Kumar, B. M. Nasipuri. "Conditional spatial fuzzy C-means clustering algorithm for segmentation of MRI images". Applied Soft Computing, vol. 34, pp. 758-769, 2015.

[19] G. S. Chuwdhury, M. Khaliluzzaman and M. Rashed-Al-Mahfuz. "MRI Segmentation using Fuzzy C-Means Clustering and Bidimensional Empirical Mode Decomposition". In: International Conference on Computer, Communication, Chemical, Materials and Electronic Engineering, 2016.

[20] E. N. Sathishkumar. "Fuzzy c Means Manual Work". Lecturer at Periyar University, Salem, 2015.

[21] R. C. Gonzalez, R. E. Woods and S. L. Eddins. "Digital Image Processing Using MATLAB". Pearson Education, London, United Kingdom, 2004. 